\title{
ML-SAI: Um Modelo Pedagógico para Atividades de M- Learning que Integra a Abordagem da Sala de Aula Invertida
}

\author{
Ernane Rosa Martins ${ }^{1}$, Luís Manuel Borges Gouveia ${ }^{2}$ \\ ${ }^{1}$ Instituto Federal de Goiás (IFG) \\ Caixa Postal 72.811-580 - Luziânia - GO - Brasil \\ ${ }^{2}$ Universidade Fernando Pessoa (UFP) \\ Caixa Postal 4249-004 - Porto - Portugal \\ ernane.martinseifg.edu.br, lmbgeufp.edu.pt
}

\begin{abstract}
This paper aims to present an analysis of the strategies of the $M L$ SAI pedagogical model, which was developed to guide m-learning activities, based on the Inverse Classroom Theory (IC). To this end, a bibliographical research was carried out on the pedagogical models, aspects related to $\mathrm{m}$ learning, the basic principles of IC and the structure and strategies of the $M L$ SAI. Next, we analyzed the results obtained with the experimentation of the model, in the discipline of introduction to programming, promoting some reflections and considerations about the same. These analyzes revealed that the model collaborates positively with the acquisition of knowledge and skills.
\end{abstract}

Resumo. Este presente trabalho tem como objetivo apresentar uma análise das estratégias do modelo pedagógico ML-SAI, o qual foi desenvolvido para orientar atividades de m-learning, fundamentado na Teoria da Sala de Aula Invertida (SAI). Para tal foi realizada uma pesquisa bibliográfica sobre os modelos pedagógicos, os aspectos relacionados à m-learning, os princípios básicos da SAI e apresenta-se a estrutura e estratégias do ML-SAI. A seguir, analisou-se os resultados encontrados com a experimentação do modelo, na disciplina de introdução a programação, promovendo algumas reflexões e considerações sobre o mesmo. Estas analises revelaram, que o modelo, colabora positivamente com a aquisição de conhecimentos e competências.

\section{Introdução}

As tecnologias digitais fazem parte da vida dos jovens atualmente, o que justifica inúmeros estudos sobre estes recursos em sala de aula, principalmente estudos que estabeleçam diretrizes para sua utilização em vista dos objetivos estabelecidos, e que abrem caminho para novas abordagens no processo de ensino e aprendizagem (Duda \& Rutz da Silva, 2015). De Bona \& Paravisi (2016) complementam que as tecnologias digitais são amplamente utilizada na atualidade por que são gratuitas e de rápido acesso à comunicação. 
Moran (2012, p. 9) argumenta que para os jovens da contemporaneidade, o mundo virtual e físico se completam, tendo uma interação cada vez mais significativa, integrando-se de forma inseparável. Para Martins da Silva, Sampaio Lima \& Bandeira Andriola (2016, p. 90), "os futuros professores devem ser preparados para enfrentar os desafios atuais de uma sociedade em constante mudança". Assim, para os mesmos autores a busca por inovação requer a adoção de práticas de ensino inovadoras, com uma abordagem mais dinâmica em sala de aula em relação aos conteúdos.

Para Moran \& Bacich (2018) as metodologias ativas tais como a sala de aula invertida, valoriza a participação efetiva dos alunos na construção do conhecimento e no desenvolvimento de suas competências, possibilita que estes aprendam em seu próprio ritmo, tempo e estilo, por meio de diferentes formas e compartilhamentos, fora e dentro da sala de aula.

O método tradicional de ensino, é geralmente realizado por meio da explanação dos conteúdos e complementada com os trabalhos de casa, entretanto na sala de aula invertida esse processo é invertido, sendo que o aluno acessa o conteúdo on-line previamente em casa antes das aulas e os momentos presenciais em sala de aula são utilizados para realizar atividades e trabalhos colaborativos pertinentes ao que foi visto em casa, por meio da interação e colaboração com seus pares e o professor. O professor fica responsável por retirar pontuais dúvidas e consolidar o aprendizado (Pereira \& Silva, 2018; Tucker, 2012). Neste sentido, Zainuddin \& Halili (2016) compilaram diferentes estudos internacionais sobre sala de aula invertida com estudantes universitários. Onde, observaram diversos benefícios, tais como: motivação, envolvimento, participação e interação.

A educação atual apresenta-se na terceira onda tecnológica denominada de Mobile Learning (m-learning), caracterizada pelo uso de equipamentos portáteis, pela mobilidade global do usuário, conectividade ubíqua, independência de dispositivo e ambiente computacional disponível em qualquer lugar e a qualquer tempo. Sendo adotado tanto para o contexto a distância como o presencial, pois o processo de ensino e aprendizagem ocorre enquanto o aluno está em sala de aula, assim como, quando está fora dela (Barcelos, Tarouco \& Berch, 2009).

Sendo assim, este artigo tem como objetivo apresentar uma análise das estratégias do modelo pedagógico ML-SAI, o qual foi desenvolvido para orientar atividades de m-learning, fundamentado na Teoria da Sala de Aula Invertida (SAI). A partir de sua experimentação, em uma disciplina de introdução a programação, de modo a possibilitar promover algumas reflexões e considerações sobre o mesmo. Tendo em vista o objetivo proposto, na seção 2 é definido o que este estudo entende por modelo pedagógico, são discutidos os aspectos relacionados à m-learning, são apresentados os princípios básicos da Teoria SAI e a estrutura e estratégias do ML-SAI. A seção 3 traz a metodologia adotada. $\mathrm{Na}$ seção 4 são apresentados as análises dos resultados encontrados com a aplicação do modelo, promovendo reflexões e considerações sobre o mesmo. Finalizando, a seção 5 apresenta algumas considerações finais sobre o presente trabalho. 


\section{Revisão Teórica}

Nesta seção são contextualizados os modelos pedagógicos, os aspectos relacionados à m-learning, os princípios básicos da SAI e são apresentados a estrutura e as estratégias do ML-SAI.

\subsection{Modelos Pedagógicos}

Para Behar, Passerino \& Bernardi (2007, p.3) modelo "é um sistema figurativo que reproduz a realidade de forma mais abstrata, quase esquemática, e que serve de referência". Os modelos pedagógicos por sua vez, são apresentados na literatura como sinônimos de estratégias de ensino, metodologias de ensino, currículo ou como teoria de aprendizagem. Assim, neste estudo, utiliza-se a definição de modelo pedagógico como sendo "um sistema de premissas teóricas que representa, explica e orienta a forma como se aborda o currículo e que se concretiza nas práticas pedagógicas e nas interações professor-aluno-objeto de conhecimento", podendo ser embasado por uma ou várias teorias de aprendizagem, ou suas reinterpretações, discutindo-se este assunto conforme os autores que adotam linhas semelhantes (Behar; Passerino \& Bernardi, 2007, p. 4).

Para a construção de um modelo pedagógico segundo Behar (2009), é necessário definir seus elementos. O modelo pedagógico deve estar baseado em duas ou mais teorias educacionais utilizadas como eixo norteador da aprendizagem e nas experiências pessoais, criando o que ela denomina de Modelo Pessoal. Conforme esta mesma autora este é fundamentado em dois elementos: Arquitetura Pedagógica (AP) e Estratégias para a Aplicação da mesma. A AP é a estrutura principal do modelo e as estratégias para a aplicação da AP são as dinâmicas do modelo pedagógico, que dependem do contexto e variáveis que envolvem o processo educativo. Estas ações didáticas direcionam o professor para colocar em pratica a AP a um contexto particular, tornando-a individualizada. A arquitetura pedagógica (AP) é constituída por:

- Aspectos organizacionais, que diz respeito a fundamentação do planejamento ou proposta pedagógica onde estão incluídos os propósitos da aprendizagem, organização do tempo e do espaço e expectativas na relação da atuação dos participantes ou da organização social da classe;

- Aspectos relacionados ao conteúdo, que engloba materiais instrucionais e/ou recursos informáticos utilizados, tais como: objetos de aprendizagem, software e outras ferramentas de aprendizagem;

- Aspectos metodológicos, que envolvem: atividades, interações, procedimentos de avaliação e a organização de todos esses elementos numa sequência didática para a aprendizagem;

- Aspectos tecnológicos, com a definição da plataforma e suas funcionalidades, ferramentas de comunicação, entre outros.

Para desenvolver um projeto pedagógico eficiente é necessário levar em consideração aspectos importantes da mudança paradigmática, principalmente referente a modalidade de ensino m-learning. 


\subsection{M-learning}

Mobile Learning (m-learning) é definido como sendo o ensino através de aparelhos móveis ou dispositivos móveis, possibilitado através dos avanços da tecnologia na área da informática. Os alunos em grande maioria possuem e estão familiarizados com o uso de dispositivos móveis, assim, estão sendo construídos sistemas educacionais voltados para serem utilizados neste tipo de dispositivo ou sendo adaptados, porém, precisam levar em conta as peculiaridades dos dispositivos produzidos por diferentes fabricantes. Este conceito permite aos alunos ter uma utilização mais flexível e otimizada do tempo, pois podem usar as tecnologias sob demanda, em qualquer lugar, em qualquer momento, ampliando a possibilidade de uso educacional de recursos que já estão sendo utilizados para comunicação e lazer. Os dispositivos móveis possuem muito potencial, permitindo oferecer diferentes níveis de envolvimento, melhorando o desempenho dos estudantes, pela colaboração ou mesmo pelos diversos formatos de materiais disponíveis, como vídeos, imagens, áudios e texto (Barcelos, Tarouco \& Berch, 2009).

Sendo assim, no m-learning o aprendizado pode ocorrer em sala de aula e em qualquer lugar no qual o estudante possa utilizar a tecnologia que já está em suas mãos para criar um novo momento de aprendizagem. Estas tecnologias podem apresentar benefícios tanto para os alunos quanto para os professores, em relação aos alunos permitem flexibilidade na aprendizagem, podendo aprender como e quando desejarem, mesmo em movimento, enquanto para aos professores é oferecido um meio de facilitar a disponibilização de materiais pedagógicos e realizar interações com os alunos (Franciscato \& Medina, 2008).

\subsection{Sala de Aula Invertida}

Para Bergmann \& Sams (2016) a Sala de Aula Invertida (SAI) é uma metodologia ativa que defende o estudo por parte dos alunos antes das aulas, através de materiais disponibilizados pelo professor, depois da realização desta interação, em sala de aula os alunos tem a oportunidade de retirar suas dúvidas, corrigir erros e aprimorar pontos importantes sobre o tema abordado (Bergmann \& Sams, 2016). Segundo os mesmos autores a inversão da sala de aula se justifica por ser durante as atividades que os alunos possuem mais dúvidas, sendo o momento ideal para realizar uma troca mais rica de conhecimentos entre o professor e os alunos. Para Valente (2014) a sala de aula tem papel fundamental dentro da metodologia da sala de aula invertida, porque é o momento em que o professor está observando e auxiliando nas atividades, realizando feedbacks constantes e contribuindo significativamente com o aprendizado dos alunos por meio de orientações.

Para Santos \& Tezani (2018) a sala de aula invertida potencializa, personaliza e facilita a aprendizagem dos alunos, atribuindo mais relevância ao aprendizado e contribuindo para aprimorar as antigas práticas pedagógicas. $\mathrm{O}$ aluno neste contexto passa a ser protagonista no processo de aprendizagem, estimulando a colaboração entre os alunos e com o professor, enquanto o professor passa a atuar como mediador no processo de aprendizagem. Conforme Oliveira \& Mendonça (2018) na metodologia da sala de aula invertida podem ser utilizados diversos recursos de modo a aproveitar a afinidade que os alunos normalmente apresentam com as tecnologias, tais como: canais do YouTube, ambientes virtuais, aplicativos de mensagens instantâneas e redes sociais. 
A teoria da sala de aula invertida é apresentada positivamente em inúmeros trabalhos investigados, tais como: Leite, Hoji \& Abdalla Junior (2018) que descreveram os desafios para a implementação do modelo de sala de aula invertida em disciplinas de Redes de Computadores em um curso técnico. Oliveira et al. (2017) que propuseram uma série de análises sobre a aplicação da metodologia em disciplinas de programação em C. Medeiros \& Bessa (2017) que propuseram uma ferramenta para análise de avaliações em grandes turmas. Santos et al. (2017), que relataram resultados positivos com a utilização do modelo no ensino médio. Chen (2014) que descreveram melhoria nas notas finais dos estudantes após aplicação do modelo no ensino de programação em $\mathrm{C}$ em um curso superior. Diferente destes, o presente trabalho buscou formatar um modelo pedagógico para contribuir com orientações no planejamento e na realização de ações de m-learning por professores e pesquisadores de diferentes áreas, tendo como base a metodologia da sala de aula invertida.

\subsection{Apresentação do ML-SAI}

Esta seção apresenta o modelo pedagógico construído a partir dos estudos preliminares (pesquisa exploratória com estudos de casos) em conjunto com a revisão bibliográfica realizada. Os estudos de caso exploratórios preliminares, utilizaram os conceitos de sala de aula invertida combinada com alguma tecnologia móvel, como Kahoot, WhatsApp e Facebook. Estes estudos de caráter exploratórios investigaram as possibilidades e potencialidades da utilização da Teoria da Sala de Aula Invertida com o auxílio de Tecnologia Móvel.

Assim, como resultados encontrados nestes estudos preliminares, destaque para: limitações e dificuldades de ordem financeira e técnica que podem excluir alguns alunos que não dispõem de smartphones, planos de internet em seus celulares ou mesmo internet em suas residências, dificultando o uso e principalmente o acesso dos alunos às ferramentas e aos recursos digitais disponíveis; benefícios significativos como o baixo custo, a acessibilidade, a interatividade e a aprendizagem colaborativa; a mediação pelo professor é fundamental, propondo temas e estimulando a participação dos alunos, identificando o contexto da sala de aula, dos alunos e da turma, estabelecendo regras e normas para utilização dos dispositivos móveis, deixando claro os objetivos e motivos das atividades propostas, verificando as limitações relevantes e os recursos tecnológicos necessários que serão utilizados, assim como, os papeis do professor e dos alunos neste processo; e por fim, é fundamental um planejamento bem estruturado por parte do professor.

O modelo pedagógico foi formatado para fornecer algumas sugestões de estratégias a professores e pesquisadores interessados em utiliza-lo, orientando estes no desenvolvimento das atividades de m-learning. Neste estudo a AP foi reestruturada e fundamentada levando em consideração os conceitos da Sala de Aula Invertida, os aspectos relacionados a utilização dos dispositivos móveis e os estudos exploratórios preliminares realizados, a AP foi estabelecida em seis aspectos, sendo estes: contexto, normatização, papeis, tecnologias, ações e limitações. A AP e as estratégias para a Aplicação da AP definidas para as atividades de m-learning estão apresentadas na Tabela 1. 
Tabela 1. Modelo Pedagógico proposto: ML-SAI

\begin{tabular}{|c|c|}
\hline $\begin{array}{c}\text { Arquitetura } \\
\text { Pedagógica (AP) }\end{array}$ & Estratégias para a Aplicação da AP \\
\hline Contexto & $\begin{array}{l}\text { - Definir os objetivos e motivos das atividades e ações proposta, deixando-os claros } \\
\text { para todos os envolvidos; } \\
\text {-Identificar os instrumentos, recursos, características das atividades e ações, dos } \\
\text { alunos e do curso; }\end{array}$ \\
\hline Normatização & $\begin{array}{l}\text { - Organizar regras e procedimentos para orientar as ações e interações; } \\
\text { - Estabelecer normas para utilização dos dispositivos móveis (quando utilizar, qual a } \\
\text { finalidade, etc.); }\end{array}$ \\
\hline Papeis & $\begin{array}{l}\text { - Compreender o papel do aluno no processo de aprendizagem, suas motivações, } \\
\text { interesses e habilidades; } \\
\text {-Entender o papel do professor como condutor e facilitador da aprendizagem; }\end{array}$ \\
\hline Tecnologias & $\begin{array}{l}\text { - Definir os dispositivos móveis, aplicativos e recursos tecnológicos que serão } \\
\text { utilizados, considerando as características físicas, técnicas e funcionais dos } \\
\text { mesmos, tais como: ambiente virtual, Sílabe, Moodle, Facebook, Khan } \\
\text { Academy, YouTube, vídeo-aula, músicas, slides, fotografias, áudios, textos, } \\
\text { entre outros, estabelecendo prioridade para aplicativos livres e gratuitos; } \\
\text {-Verificar a necessidade e disponibilidade de conexão com a Internet; }\end{array}$ \\
\hline Ações & $\begin{array}{l}\text {-Especificar se as ações serão individuais, em grupo ou ambas, se estas serão } \\
\text { comuns a todos os alunos ou diferenciadas por aluno ou grupo de alunos; } \\
\text {-Definir ferramentas de comunicação e sistemas de apoio para dar suporte aos } \\
\text { alunos em caso de dificuldades; } \\
\text {-Definir se as ações serão realizadas em um mesmo local, ao mesmo tempo ou em } \\
\text { locais e momentos distintos; } \\
\text { •Estabelecer práticas educacionais favoráveis ao aprendizado (situações problemas, } \\
\text { aplicações práticas, colaborativas, autônomas, críticas, em contextos reais, } \\
\text { pesquisas), levando em consideração os ambientes de aprendizagem (on-line, } \\
\text { salas de aula, laboratórios) de preferência com os dispositivos móveis dos } \\
\text { próprios alunos; } \\
\text {-Incentivar a interação entre os alunos e com o professor, por meio do uso de } \\
\text { dispositivos móveis, com foco no desenvolvimento da atividade proposta; } \\
\text {-Determinar os mecanismos de avaliação de desempenho e certificação da } \\
\text { aprendizagem, se individuais ou em equipes, de preferência continua, e } \\
\text { disponibiliza-los para os alunos; } \\
\text { •Estabelecer momentos de reflexão e análise das atividades realizadas, buscando } \\
\text { colaborar na melhoria continua de novas atividades; } \\
\text { •Estruturar os conteúdos que serão disponibilizados em ambiente virtual, para que } \\
\text { os alunos possam acessa-los por meio de um dispositivo móvel, quando e } \\
\text { quantas vezes quiserem, se possível com o acompanhamento das visualizações } \\
\text { pelo professor; } \\
\text { • Realizar uma curadoria dos conteúdos já existentes na internet, por meio de } \\
\text { plataformas como Khan Academy e o YouTube em busca de bons vídeos } \\
\text { educativos, ou caso não sejam encontrados, gravar vídeos ou áudios utilizando } \\
\text { as ferramentas que existem no próprio dispositivo móvel; } \\
\text { •Estimular diferentes formas de aprendizado por meio de diferentes fontes de } \\
\text { conteúdo, tais como: vídeos, áudios, imagens, textos, slides, questões, entre } \\
\text { outras; } \\
\text { a otimizar o tempo em sala de aula, utilizando projetos, trabalhos ou solução de } \\
\text { problemas, que se conectem com o que foi visto previamente na plataforma; }\end{array}$ \\
\hline Limitações & $\begin{array}{l}\text { - Levantar os principais pré-requisitos das atividades e possíveis distratores do } \\
\text { aprendizado; } \\
\text {-Identificar quais conteúdos podem ser melhor trabalhados com tecnologias } \\
\text { móveis; }\end{array}$ \\
\hline
\end{tabular}


-Verificar se os materiais pedagógicos podem ser utilizados em dispositivos móveis, considerando tamanho da tela, usabilidade, capacidade de armazenamento e modelos de dispositivos diferentes;

- Verificar a disponibilidade de dispositivos móveis, tomadas para recarregar as baterias dos celulares, conexão com a Internet, quando necessário, e se os aplicativos apresentam interface adequada a aprendizagem do conteúdo;

O modelo pedagógico apresentado não é uma estrutura inflexível, visto que pode se adaptar e colaborar com o desenvolvimento de diversas atividades de m-learning envolvendo diferentes conteúdos e dispositivos móveis.

\section{Metodologia}

A presente pesquisa caracteriza-se como exploratória. De acordo com Gil (1999), a pesquisa exploratória visa desenvolver, esclarecer ou modificar conceitos e ideias sobre determinado assunto. A investigação realizada é de natureza exploratória e descritiva, considerando que as pesquisas sobre o fenómeno investigado são novas e encontram-se em uma fase inicial. Os procedimentos metodológicos, consistiram em: observação dos recursos tecnológicos, das atividades, dos conteúdos, dos papeis dos alunos e professor, da troca de conhecimento, da responsabilidade e autonomia dos estudantes e diários utilizados pelo professor.

O modelo pedagógico descrito foi experimentado durante o segundo semestre de 2018, na disciplina de introdução a programação, de um curso técnico presencial de informática. O dispositivo móvel adotado foi o celular dos próprios alunos e o ambiente de aprendizagem utilizado para disponibilizar os conteúdos em forma de vídeos, slides, textos e links, foi o edmodo, por ser o que os alunos já estavam familiarizados em outras disciplinas e por contar com uma versão de aplicativo para dispositivos móveis.

\section{Aplicação do ML-SAI: Analises e Reflexões}

Apesar da impossibilidade de realizar a aplicação do modelo em todas as unidades didáticas da disciplina, foram observados dois principais tipos de resultados positivos em relação ao aproveitamento e participação dos alunos.

O primeiro resultado observado foi uma postura de maior responsabilidade no estudo da temática de cada unidade. Por não terem os conteúdos apresentados em detalhes na forma tradicional como uma palestra, os alunos precisam estudar por conta própria buscando tirar suas dúvidas com outros colegas ou com o próprio professor no encontro presencial em sala de aula. Como ficou estabelecido que os alunos iriam realizar apresentações sobre os conteúdos estudados, eles sempre buscavam aprimorar os estudos por conta própria.

O segundo aspecto observado foi o maior grau de envolvimento e dedicação nos projetos realizados. Apesar da necessidade de dedicar mais tempo na realização dos projetos, os resultados e benefícios do esforço maior ficou evidente para os alunos, principalmente por ter gerado mais interesse por parte dos mesmos.

Um exemplo de projeto realizado na disciplina de introdução a programação foi a elaboração de um programa de cálculo de matrizes. Foram realizadas as quatro operações matemáticas básicas: somo, subtração, multiplicação e divisão de matrizes. $\mathrm{O}$ desempenho dos alunos neste projeto foi considerado por toda a turma entre bom e 
excelente, sendo que eles aprenderam as estruturas das matrizes conforme a linguagem de programação por meio de vídeo antes das aulas e identificaram as possíveis soluções para o problema em sala de aula, todos os alunos criaram algum tipo de implementação, ainda que parcial para o problema, mas pelo fato do aprendizado e o desenvolvimento do trabalho ser totalmente independente da ajuda do professor, os resultados como um todo foram impressionantes.

Nesse projeto sobre cálculo de matrizes, os alunos foram orientados a pensar em um problema real o que despertou o interesse dos alunos, visto que esta aplicação poderia ser disponibilizada e utilizada posteriormente para realizar este tipo de cálculo quando necessário. Mesmo sendo um projeto inicial de programação, a intenção é motivar os alunos a realizarem algo útil, assim adotou-se sempre esta estratégia nos demais projetos da disciplina.

Outro aspecto interessante que merece destacar é a possibilidade de realizar projetos integrando os conteúdos da computação aos conceitos de outra disciplina, neste caso com a matemática. Para a realização do projeto, os alunos tiveram que aprender e dominar os conceitos de lógica de programação, entender como empregar a linguagem de programação e conhecimentos de matemática, aprimorando sempre os conhecimentos disponibilizados em vídeos com pesquisas adicionais, principalmente por meio da internet.

Um segundo exemplo de projeto realizado foi a implementação de uma calculadora de massa corporal. Neste projeto os alunos precisaram pesquisar como determinar a massa corporal de homens e mulheres. Os smartphones antes utilizados para atividades sem importância, agora tornaram-se instrumentos imprescindíveis para realizar as pesquisar e assim facilitar o aprendizado. Mesmo os alunos relatando que precisaram dedicar grande quantidade de tempo para a realização dos projetos, os mesmos reconheceram que os benefícios foram evidentes em termos de aprendizagem do conteúdo da disciplina e crescimento pessoal dos alunos.

Outro aspecto observado em relação aos vídeos foi a importância de utilizar materiais multimídias atraentes para os alunos. Assim como no trabalho de Schmidt e Ralph (2016) foi detectado que os alunos expressam desprazer e tédio com o conteúdo dos vídeos. Segundo estes mesmos autores, é importante desenvolver e usar os materiais multimídia corretamente. Os vídeos precisam ser breves, precisos e agradáveis, eles têm que dividir o assunto de maneira coerente e, assim, facilitar a internalização de seu conteúdo.

Ao final o professor alegou que a experiência foi positiva, apesar de apresentar prós e contras, em relação ao aspecto positivo foi registrado a excelente aceitação por parte dos alunos, mas em contraponto negativo, foram registrados alguns apontamentos de dificuldade de acesso aos conteúdos online, justificados pela falta de tempo, o que impactou no estudo prévio por parte de alguns alunos em relação ao conteúdo, gerando maior dificuldade em sala de aula, forçando o professor a apresentar o conteúdo nos moldes do ensino tradicional. É importante destacar que os alunos participantes desta pesquisa nunca haviam entrado em contato com nenhuma proposta de modelo pedagógico como o apresentado neste trabalho ou nem mesmo com a metodologia da sala de aula invertida. 


\section{Considerações Finais}

Este trabalho tem como objetivo apresentar uma análise das estratégias do modelo pedagógico ML-SAI, o qual foi desenvolvido para orientar atividades de m-learning, tendo como fundamentação a SAI. A experimentação do modelo, na disciplina de introdução a programação, possibilitou promover algumas reflexões e considerações importantes sobre o mesmo.

Assim, analisando a experimentação realizada, foi possível observar pelo desenvolvimento das atividades que o modelo pedagógico utilizado aparentemente ajudou os alunos a assumir maior responsabilidade no processo de aprendizagem. Além de contribuir para diminuir a quantidade de aulas expositivas que tornam as mesmas cansativas, ajudar com que os alunos busquem entender o processo para alcançar uma determinada solução de problema ao invés de decorar passos determinados, aprimorar o raciocínio e a reflexão buscando explorar o potencial do uso prático da programação em contextos reais. Mesmo assim, apesar de aparentemente contribuir fortemente com o desenvolvimento de habilidade na solução de problemas, é necessário a realização de estudos adicionais para confirmar tal fato.

O modelo permitiu elaborar projetos de interesses dos alunos, com resultados mais concretos, o que motivou os mesmos e permitiu também maior integração de conteúdos, com uma abordagem mais prática, integrando a programação com matérias, tais como: física, química e matemática. Posteriormente, pretende-se realizar novas experimentações, de modo a melhorar e consolidar o modelo pedagógico desenvolvido. Posteriormente, o mesmo será divulgado e disponibilizado em forma digital por meio de aplicativo móvel, de modo a possibilitar a sua ampla utilização.

\section{Referencias}

Barcelos, R. J. S.; Tarouco, L. and Berch, M. (2009). O uso de mobile learning no ensino de algoritmos. Revista Novas Tecnologias na Educação, Porto Alegre, v. 7, n. 2, p. 1-11.

Behar, P. A. (2009). Modelos pedagógicos em educação a distância. 311 p. Porto Alegre: Artmed.

Behar, P. A.; Passerino, L. and Bernardi, M. (2007). Modelos Pedagógicos para Educação a Distância: pressupostos teóricos para a construção de objetos de aprendizagem. Revista Novas Tecnologias na Educação, Porto Alegre, v.5, n. 2, p. 1-12.

Bergmann, J. and Sams, A. (2016). A. Sala de aula invertida: uma metodologia ativa de aprendizagem. 1 ed. Rio de Janeiro: LTC. 114 p.

Chen, E. Z. (2014). Flipped Classroom Model and Its Implementation in a Computer Programming Course. Lärarlärdom, v. 2014, p. 180-200.

De Bona, A. and Paravisi, M. (2016). O Whatsapp: um espaço de construção escolar e administrativa. Revista Thema, Pelotas, v.13, n.1, p. 15-23.

Duda, R. and Rutz Da Silva, S. De C. (2015). A tecnologia como recurso auxiliary na ressignificação do processo resolutivo de equações. Revista Tecnologias na Educação. Ano 7, n. 13, p.1-11.

Franciscato, F. T. and Medina, R. D. (2008). M-Learning e Android: um novo paradigma? Revista Novas Tecnologias na Educação, Porto Alegre, v. 6, n. 1, p. 1-10. 
Gil, A. C. (1999). Métodos e técnicas de pesquisa social. São Paulo: Atlas.

Leite, F. N.; Hoji, E. S. and Abdala Junior, H. (2018). A Blended Learning Method Applied in Data Communication and Computer Networks Subject. IEEE Latin America Transactions, v. 16, n. 1, p. 163-171.

Martins Da Silva, F. C., Sampaio Lima, A. E. and Bandeira Andriola, W. (2016). Avaliação do suporte de TDIC na formação do pedagogo: Um estudo em Universidade Brasileira. REICE. Revista Iberoamericana sobre Calidad, Eficacia y Cambio en Educación, 14(3), $77-93$.

Medeiros, R. A. C. and Bessa, A. (2017). MiniTeste: uma ferramenta ágil para aplicação de avaliações personalizadas. Revista Novas Tecnologias na Educação, Porto Alegre, v. 15, n. 1, p. 1-10.

Moran, J. E. and Bacich, L. (2017). Metodologias ativas para uma educação inovadora: Uma abordagem teórico-prática. $1^{\mathrm{a}}$ ed. Porto Alegre: Penso. 260 p.

Moran, J. M. (2012). A Educação que Desejamos: Novos desafios e como chegar lá. $5^{a}$ ed. Campinas, São Paulo: Papirus.

Oliveira, M. G. et al. (2017). Um Curso de Programação a Distância com Metodologias Ativas e Análise de Aprendizagem por Métricas de Software. Revista Novas Tecnologias na Educação, Porto Alegre, v. 15, n. 1, p. 1-10.

Oliveira, S. A. B. and Mendonça, A. P. (2018). Sala de Aula Invertida - Uma Experiência no Ensino-Aprendizagem de Programação para Administração de Redes de Computadores. Revista Novas Tecnologias na Educação, Porto Alegre, v. 16, n. 1.

Pereira, Z. T. G. and Silva, D. Q. (2018). Metodologia Ativa: Sala de Aula Invertida e suas Práticas na Educação Básica. EICE. Revista Iberoamericana sobre Calidad, Eficacia y Cambio en Educación, 16(4), p. 63-78.

Santos, A. C. et al. (2017). Ensino Híbrido: Relato de Experiência sobre o uso de AVEA em uma proposta de Sala de Aula Invertida para o Ensino Médio. Revista Novas Tecnologias na Educação, Porto Alegre, v. 15, n. 2, p. 1-10.

Santos, L. F. and Tezani, T. C. R. (2018). Aprendizagem Colaborativa no Ensino de História: A Sala de Aula Invertida como Metodologia Ativa? Revista Novas Tecnologias na Educação, Porto Alegre, v. 16, n. 2. p. 1-10.

Schmidt, S. Y. and Ralph, D. (2016). The flipped classroom: a twist on teaching. Contemporary Issues in Education Research (Online), v. 9(1), p. 1-6.

Tucker, B. (2012). The flipped classroom. Education Next, 12(1), p. 82-83.

Valente, J. A. (2014). Blended Learning e as Mudanças no Ensino Superior: a Proposta da Sala de Aula Invertida. In Educar em Revista, Curitiba, PR, Edição Especial, n. 4, p. 79 97, Editora UFPR.

Zainuddin, Z. Y. and Halili, S. H. (2016). Flipped classroom research and trends from different fields of study. The International Review of Research in Open and Distributed Learning, v. 17(3), p. 313-340. 